\title{
Multiple sclerosis presenting with acute remitting psychiatric symptoms
}

\author{
W. B. M A T T H E W S \\ From the University Department of Clinical Neurology, Churchill Hospital, Oxford
}

SUMMARY Two patients are described in whom acute symptoms of apparently primary psychiatric disease could be diagnosed in retrospect as due to multiple sclerosis. In both patients the initial symptoms recovered completely. In a third patient, also presenting with mental symptoms, this diagnosis would not have been suspected on clinical grounds but is suggested by the results of modern diagnostic techniques.

Persistent mental symptoms, predominantly those of dementia, are fully documented in multiple sclerosis, even occasionally from the early stages of the disease (Cottrell and Wilson, 1926; O'Malley, 1966; Surridge, 1969; Young et al., 1976). It is less well known that multiple sclerosis may present as an acute psychiatric problem with subsequent complete remission. Two such cases are described in whom later developments leave little doubt that the initial symptoms were caused by multiple sclerosis. In a third patient this diagnosis remains uncertain and would scarcely have been entertained without the contribution of modern diagnostic methods.

\section{Case reports}

CASE 1

An unmarried girl (PS), aged 19 years, was in good health until on 9 April 1972 she was noticed to be speaking repetitively and unduly rapidly. In the next few days she was intermittently confused and her speech was slurred. She refused all food, and by 16 April she was lying down all day, was incontinent of urine, and hardly speaking. She was admitted on 18 April to the Royal Berkshire Hospital (Dr E. V. Cox). At this time she was mute but otherwise no abnormal neurological signs were found. Her behaviour became increasingly bizarre with episodes of cycling movements of the legs, rocking of the body, or repeated banging of the head on the pillow. She was transferred to Fairmile Mental Hospital (Dr A. A.

Address for reprint requests: Professor W. B. Matthews, Department of Clinical Neurology, The Churchill Hospital, Headington, Oxford OX3 7LJ.

Accepted 10 March 1979
Salama) where schizophrenia was thought to be the probable diagnosis. Treatment with chlorpromazine brought no improvement and she therefore received electroconvulsant therapy on 29 April, 1 and 5 May, but without benefit. On 7 May she had four major epileptic fits and two more the next day. She was transferred to the Neurology Department at the Churchill Hospital.

She was found to be awake and apparently aware of her surroundings. She moved her eyes and limbs spontaneously and in response to command and could stand unaided, but did not speak at all and did not withdraw from painful stimuli. Otherwise examination was entirely normal. She was not febrile.

At lumbar puncture the CSF pressure was normal. The total protein was $0.25 \mathrm{~g} / 1$ and there were 15 lymphocytes $/ \mathrm{mm}^{3}$. This examination was repeated on 15 May as it was thought possible that the repeated fits might be the cause of the pleocytosis. The protein was then $0.4 \mathrm{~g} / 1$ and there were 21 lymphocytes $/ \mathrm{mm}^{3}$. No Lange curve was performed and the specimen sent for IgG estimation went astray. The WR was negative, and routine viral studies showed no evidence of infection. Blood count, ESR, skull and chest radiographs, and an isotope brain scan were normal. An EEG on 9 and 16 May showed a diffuse excess of intermediate slow activity (Dr E. W. Poole).

She was given phenytoin $300 \mathrm{mg}$ a day and had no more fits. She began to improve, and by 17 May was active and occupied herself sewing but did not speak spontaneously. She was discharged home on 25 May, and when seen on 7 June her mental state was normal and there were no abnormal neurological signs. Her EEG had also reverted 
towards normality. She had no clear memory of her illness. Treatment was stopped and she returned to work as a bank clerk. No diagnosis was made.

She remained well until January 1975 when she developed blurred vision in the left eye for which she attended another hospital. A central scotoma was found and this resolved within two months. In March 1976 she awoke one morning to find that she was completely deaf in the left ear. The detailed results of otological examination are not available but apparently no peripheral lesion was found and hearing recovered within a few months. In September 1976 she developed numbness of the skin of the back and lower abdomen, spreading to the buttocks and thighs and lost the normal sensation of passing urine. When seen six weeks later she had begun to improve. The optic discs were normal. The tendon reflexes were increased in the right arm and leg but the plantar reflexes were flexor. Superficial abdominal reflexes were absent. Cutaneous sensation was blunted from $\mathrm{T} 10$ to $\mathrm{L} 4$ segments bilaterally.

Pattern reversal visual evoked potentials (VEP) showed prolonged latency from each eye: right, $123 \mathrm{~ms}$, left $154 \mathrm{~ms}$ (upper limit of normal $111 \mathrm{~ms})$.

The sensory symptoms recovered but in February 1977 both legs became weak and she was occasionally incontinent of urine. Both plantar responses were now extensor. However, she again recovered and by May 1977 was free from symptoms, the only abnormal physical sign being temporal pallor of the optic discs.

CASE 2

A married woman (DL), aged 23 years, had been well until March 1976 when she rapidly became increasingly depressed, inactive, and uncommunicative. Eventually she simply sat at the kitchen table unwilling to move or speak. She was admitted to the Seymour Psychiatric Centre (Dr M. A. T. Waters). She became increasingly withdrawn and unable to dress or care for herself. She was intermittently incontinent of urine and faeces. Chlorpromazine $150 \mathrm{mg}$ a day brought no improvement. The tendon reflexes in the lower limbs became exaggerated, and the left plantar reflex was thought to be extensor. Accordingly she was transferred to the Churchill Hospital on 6 April. There was no past history of neurological disease.

On admission she was withdrawn and retarded with expressionless facies. She admitted to depression and complete loss of appetite, and refused to answer further questions. Her upper limbs would retain for several minutes any posture in which they were placed. She did not cooperate in neurological examination. The tendon reflexes in the lower limbs were increased and the 0 plantar reflexes difficult to interpret but within a few days were bilaterally extensor. Her gait appeared to be ataxic and her trunk was held in an unusual flexed posture. A diagnosis of schizophrenia or of acute depression had been considered $\overrightarrow{\vec{F}}$ but at lumbar puncture the CSF contained $0.5 \mathrm{~g} / 1+\underset{0}{0}$ of protein and 48 lymphocytes $/ \mathrm{mm}^{3}$. The Lange curve was flat and the proportion of $\mathrm{IgG}$ was $\frac{\bar{c}}{\bar{c}}$ $13 \%$.

The patient's mental state varied from being almost normally communicative to outbursts of $\mathrm{\omega}$ loud shouting accompanied by kicking and arch- $\vec{P}$ ing of the back. Her replies to questions on general $\overrightarrow{\vec{H}}$ knowledge were often absurd-she claimed that $\tilde{o}^{\circ}$ the President of the United States was George Washington. Often she would simulate sleep to avoid examination.

Although there was no neck rigidity or fever and the blood count and ESR were normal, there of was a persistent lymphocytosis in the CSF, and 0 an extensive search was made for possible ing fection but with negative results. The only possib significant finding was a measles antibody titre of $\mathbb{C}$ $1 / 128$ in the blood and $1 / 4$ in the CSF. The CSF glucose remained normal. Tests for collageg $\frac{\pi}{3}$ disease and porphyria were negative, and pneumoencephalogram and isotope brain sca were normal. The EEG contained a marked excess $\overrightarrow{0}$ of bilateral slow activity (Dr E. W. Poole): VEPs ç were within the normal range. Psychometry o showed evidence of severe impairment of verbal and nonverbal skills but the patient undoubtedly falsified the results of many of these tests deliberately.

As she was not improving and no diagnosis had been made a biopsy was taken from the right frontal lobe (Mr M. Briggs). This showed normal cortex and underlying white matter but there was a chronic inflammatory reaction in the leptomeninges with foci of lymphocytes, extending into the Virchow-Robin spaces (Dr D. Oppenheimer).

On 12 May treatment with prednisolone $80 \mathrm{mg}$ a day was begun, gradually reducing to $15 \mathrm{mg}$ a day over a month. On 17 May the CSF contained only 3 lymphocytes $/ \mathrm{mm}^{3}$, and on 18 May the patient was alert and was speaking spontaneously. 은 On 7 June the plantar reflexes were flexor. A $>$ month later the patient was free of symptoms and mentally normal. Sensory testing was now possible $N$ and vibration sense was found to be absent at the ankles. In August 1976 steroid treatment was $\tilde{O}$ 
stopped and the patient was entirely normal. No diagnosis had been made.

The patient left the area but was seen again in June 1978. She had no symptoms but said that in September 1976 the vision of the right eye had suddenly become blurred. It was painless and vision improved but did not return to normal. The optic discs were normal. Visual acuity was $6 / 9$ in the right eye and $6 / 6$ in the left. Vibration sense was found to be absent below the hips. The VEPs were abnormal, the latency of the P100 from the right eye being $136 \mathrm{~ms}$ and from the left eye $124 \mathrm{~ms}$ (upper limit of normal $111 \mathrm{~ms}$ ).

CASE 3

A married woman of 24 years (AT) was admitted to the Neurosurgical Department of the Radcliffe Infirmary under the care of $\mathrm{Mr} \mathrm{J}$. Pennybacker in March 1971. Four weeks previously she had undergone a spontaneous abortion of a three month gestation. A week later she had an episode of vomiting and became lethargic. She lay in bed most of the time and was mentally confused. She was admitted to another hospital where she was found to have bilateral papilloedema. Despite this, a lumbar puncture was performed, the pressure being recorded as $180 \mathrm{~mm}$ of water. The CSF total protein was $0.8 \mathrm{~g} / 1$ and there was 1 lymphocyte per $\mathrm{mm}^{3}$.

On admission she was found to be disorientated in time and place. Her affect was flat and she appeared to have no insight into her condition. She was not dysphasic. There was a moderate degree of bilateral papilloedema with a few small haemorrhages around the discs. Visual acuity could not be tested accurately but was not thought to be greatly reduced. She did not cooperate in neurological examination but it was thought that there was some weakness of the right upper limb with no other abnormality. A left carotid angiogram was normal. The EEG contained a bilateral excess of slow frequencies. Lumbar puncture was repeated and again showed no increase in pressure. The total protein was $0.6 \mathrm{~g} / 1$ with no increase in the proportion of IgG. There were 5 lymphocytes/ $\mathrm{mm}^{3}$. The WR was negative, and the chest radiograph was normal. She was transferred to the Neurological Department.

Over the next three weeks she became much more alert but was unable to find her way about the ward. The papilloedema gradually subsided and visual acuity was recorded as $\mathrm{R} 6 / 9, \mathrm{~L} 6 / 12$ a month after her admission. Her verbal IQ at this time was assessed as 88 and performance as 51. There were no signs of focal neurological disease. Improvement continued, and she became excessively talkative, disinhibited, and flirtatious. She was discharged home, and in June 1971 was considered by a psychiatrist to be hypomanic. However, in October 1971 she appeared to be physically and mentally normal. Her verbal IQ was 102 and performance 90, figures rather below those to be expected from her school career. At this time she had no memory of the early weeks in hospital. She attributed her illness to the deliberate inhalation of hair lacquer which she claimed to have done several times a day for three years in order to conceal the smell of tobacco from her husband who disapproved of smoking.

She was not seen again until April 1978 when she was readmitted for the investigation of epilepsy. It proved difficult to unravel the details of her intervening history, partly because one of her major complaints was of memory failure. In 1972 she had received treatment for "hypomania", and in 1973 developed infrequent major epileptic fits, apparently followed by transient weakness of the right side. Her father thought that she had never fully recovered from the initial illness and stated that in October 1977 there had been a noticeable deterioration in her condition. Her writing became illegible. She began to lose her way in familiar surroundings and had difficulty in managing her 8 year old daughter and her home. She had recently divorced her husband.

On examination the immediate impression was that she was fabricating or exaggerating her disabilities. She talked in a childish and inconsequential way and her apparent memory failure was fluctuating and inconsistent. The optic fundi were normal. There were frequent irregular jerking movements of the right arm, aggravated by attempted voluntary movement and greatly reduced when her attention was distracted. The plantar reflexes were flexor. Sensory testing was unreliable. However, she was unquestionably suffering from organic nervous disease. Psychometry showed intellectual impairment with particular emphasis on memory function. The EEG had deteriorated from the first recording in 1971 and showed a considerable excess of slow activity, more marked on the left side. The CAT scan (Figure) showed moderate symmetrical ventricular dilatation and low density periventricular flares (Dr Philip Sheldon). Despite normal visual acuity, VEPs were markedly delayed from both eyes-144 ms from the right and 141 from the left (upper limit of normal $111 \mathrm{~ms}$ ). The CSF was normal, $0.5 \mathrm{~g} / 1$ of protein $(13 \% \mathrm{IgG})$ and 4 lymphocytes $/ \mathrm{mm}^{3}$.

After her discharge from hospital, the patient has had no further fits on sodium valproate $400 \mathrm{mg}$ 

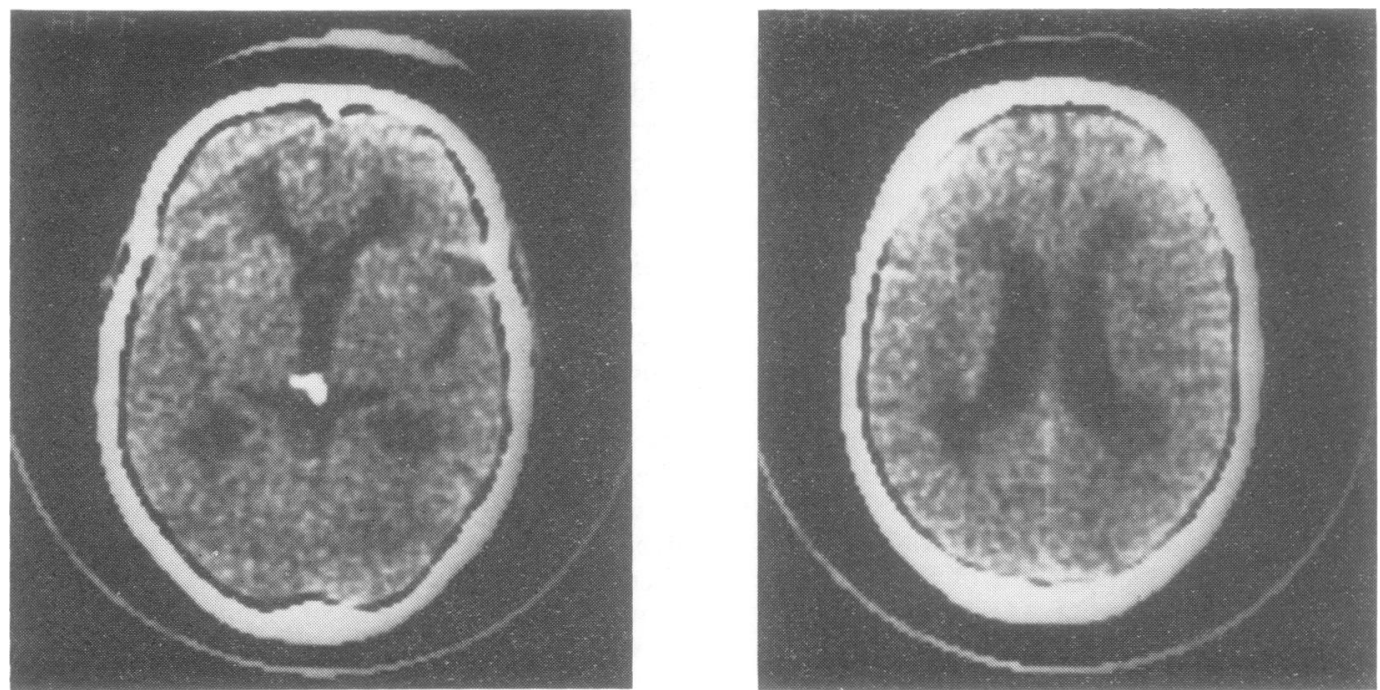

Figure CAT scans of case 3 showing symmetrical ventricular dilatation and low density periventricular flares.

thrice daily. Her intellectual capacity has been adequate for her to care for her child at home with some parental assistance. Involuntary movements of the right arm persist.

\section{Discussion}

The first patient described subsequently developed clinically definite multiple sclerosis, and there must be a strong presumption that the presenting illness was also caused by this disease. There were no findings on physical examination that could have pointed to this diagnosis. The lymphocytosis in the CSF, while impossible to interpret at the time, also indicates that the initial symptoms were those of organic nervous disease. Epilepsy occurring during a relapse or first episode of multiple sclerosis without later recurrence has been described previously (Matthews, 1962). In the second patient the extensor plantar reflexes eventually indicated the organic nature of the initial illness, again supported by the abnormal findings in the CSF. Recovery was followed by presumed unilateral optic neuritis and bilateral abnormality of VEP. In both these patients remission of the mental symptoms was complete.

In the third patient the diagnosis is plainly quite uncertain. Her own explanation that her illness was caused by the deliberate inhalation of hair lacquer cannot be substantiated. The habitual inhalation of aerosol, even direct spraying into the mouth, is a recognised form of drug abuse, the desired effect being derived from the pro- pellant (Hofmann and Hofmann, 1975). Theo o nature of the propellant in the present instance is unknown, and may well have been innocuous $\frac{\mathbb{\Omega}}{7}$ 의 I have not been able to trace an account of $\vec{D} \vec{c}$ encephalopathy after aerosol inhalation. The $\mathbb{D}$ grossly abnormal VEP in the presence of norma $\frac{d}{10}$ visual acuity is strong evidence in favour of demyelination. The periventricular low density. areas on the CAT scan are similar to those re윽 ported in multiple sclerosis (Cala et al., 1978): The initial mental symptoms appeared to recover but in retrospect this may not have been complete. The persistent physical abnormalities are in no way typical of multiple sclerosis.

All these patients had been regarded initially as suffering from primary mental disease, and two had been admitted to psychiatric hospitals thought to be suffering from schizophrenia. There are a number of reports of schizophrenia in patients with multiple sclerosis. Schmalzbach (1954) recorded multiple sclerosis developing in a patient with chronic schizophrenia. In Parker's (1956) patient, the onset of the two conditions was more closely related but both were chronic and progressive. Gallineck and Kalinowsky (1958) described two patients with multiple sclerosis who later developed schizophrenia which they regarded as unrelated. Geocaris (1957) reported four patients in whom psychotic episodes appeared to herald the onset of multiple sclerosis. However, all had a long history of less severe psychiatric symptoms before the onset of persistent mental disorder and overt evidence of multiple sclerosis. 
The patient described by Hollender and Steckler (1972) developed a severe mental disorder thought to resemble schizophrenia in the course of an apparently typical multifocal onset of multiple sclerosis. Both conditions recovered completely, and there had been no recurrence seven years later so that the diagnosis must remain in doubt. Targowla's (1927) patient, while certainly a typical candidate for multiple sclerosis"courageuse, sérieuse, bonne ouvrière"-was described only two months after the onset of mania followed by predominantly brainstem signs, and again the diagnosis is uncertain.

I have found no exact parallel to the first two cases reported here, that is to say, an acute illness mimicking primary psychiatric disease followed by complete remission and by later evidence of multiple sclerosis. The patient described by McAlpine et al. (1968) is, however, in many ways similar. This was a woman aged 20 years whose initial symptom was that she became completely inactive. Symptoms of organic nervous disease consisted of tingling down the left side and she was observed to have a mild left hemiparesis. However, visual and auditory hallucinations and a diffuse disturbance in the EEG indicated widespread cerebral disorder which completely recovered. The subsequent history was that of typical multiple sclerosis.

In such cases it is legitimate to attribute the mental changes to multiple sclerosis. Absence of localising signs and the diffuse nature of the EEG abnormality suggest that some widespread effect of the disease is responsible for the symptoms rather than a strategically situated plaque. Geocaris (1957) when considering his patients with both multiple sclerosis and schizophrenia sensibly found himself unable to come to a conclusion about the possible relationship of two conditions of unknown cause. Unfortunately it was not possible to obtain CAT scans during the acute phase of the initial illness in the patients described here, and it is likely that serial scans might have thrown light on the underlying cerebral pathology.

The purpose of this communication is to draw attention to the need to consider multiple sclerosis among the causes of acute mental symptoms in previously healthy young adults. The third case is described in order to demonstrate that modern diagnostic techniques may suggest the diagnosis of multiple sclerosis where it might not otherwise have been suspected.

\section{References}

Cala, L. A., Mastaglia, F. L., and Black, J. L. (1978). Computerised tomography of brain and optic nerve in multiple sclerosis-observations in 100 patients including serial studies in 16. Journal of the Neurological Sciences, 36, 411-426.

Cottrell, S. S., and Wilson, S. A. K. (1926). The affective symptomatology of multiple sclerosis. Journal of Neurology and Psychopathology, 7, 130.

Gallineck, A., and Kalinowsky, L. B. (1958). Psychiatric aspects of multiple sclerosis. Diseases of the Nervous System, 19, 77-80.

Geocaris, K. (1957). Psychotic episodes heralding the diagnosis of multiple sclerosis. Bulletin of the Menninger Clinic, 21, 107-116.

Hofmann, F. G., and Hofmann, A. D. (1975). A Handbook on Drug and Alcohol Abuse, p. 134. Oxford University Press: New York.

Hollender, M. H., and Steckler, P. P. (1972). Multiple sclerosis and schizophrenia: a case report. International Journal of Psychiatry in Medicine, 3, 251257.

McAlpine, D., Lumsden, C. E., and Acheson, E. D. (1968). Multiple Sclerosis: A Reappraisal. First edition reprint, p. 136. Churchill Livingstone: Edinburgh.

Matthews, W. B. (1962). Epilepsy and disseminated sclerosis. Quarterly Journal of Medicine, 31, 141155.

O'Malley, P. P. (1966). Severe mental symptoms in disseminated sclerosis: a neuropathological study. Journal of the Irish Medical Association, 58, 115127.

Parker, N. (1956). Disseminated sclerosis presenting as schizophrenia. Medical Journal of Australia, 1, 405-407.

Schmalzbach, O. (1954). Disseminated sclerosis in schizophrenia. Medical Journal of Australia, 1, 451-452.

Surridge, D. (1969). An investigation into some psychiatric aspects of multiple sclerosis. British Journal of Psychiatry, 115, 749-764.

Targowla, H. (1927). Sclérose en plaque fruste à début mental. L'Encephale, 22, 169-175.

Young, A. C., Saunders, J., and Ponsford, J. R. (1976). Mental change as an early feature of multiple sclerosis. Journal of Neurology, Neurosurgery, and Psychiatry, 39, 1008-1013. 